\title{
Tunable optical buffer based on III-V MEMS design
}

\author{
Wing H. Ng*a ${ }^{\mathrm{a}}$, Nina Podoliak ${ }^{\mathrm{b}}$, Peter Horak ${ }^{\mathrm{b}}$, Jiang $\mathrm{Wu}^{\mathrm{a}}$, Huiyun Liu ${ }^{\mathrm{a}}$, William J. Stewart ${ }^{\mathrm{b}}$, and \\ Anthony J. Kenyon ${ }^{\mathrm{a}}$ \\ ${ }^{a}$ Department of Electronic and Electrical Engineering, University College London, Torrington Place, \\ London WC1E 7JE, UK \\ ${ }^{\mathrm{b}}$ Optoelectronics Research Centre, University of Southampton, Highfield, Southampton SO17 1BJ, \\ UK
}

\begin{abstract}
We present the design and fabrication of a tunable optical buffer device based on III-V semiconductor platform for telecommunication applications. The device comprises two indium phosphide suspended parallel waveguides with cross sectional dimension of $200 \mathrm{~nm}$ by $300 \mathrm{~nm}$, separated by an air gap. The gap between the waveguides was designed to be adjustable by electrostatic force. Our simulation estimated that only $3 \mathrm{~V}$ is required to increase the separation distance from $50 \mathrm{~nm}$ to $500 \mathrm{~nm}$; this translates to a change in the propagation delay by a factor of 2 . The first generation of the suspended waveguide structure for optical buffering was fabricated. The sample was grown on an InP substrate by molecular beam epitaxy. The waveguide pattern is written onto a $300 \mathrm{~nm}$ thick $\mathrm{InP}$ device layer by electron beam lithography and plasma etching. Electrodes were incorporated into the structure to apply voltages for MEMS actuation.
\end{abstract}

Keywords: Optical buffer, waveguide, III-V semiconductors, MEMS, MOEMS, optical delay, actuation.

\section{INTRODUCTION}

An optical buffer is a very important component in realizing all-optical telecommunication networks. Its functionality is to temporarily store light and adjust the timing of the optical signal travelling through the network. There are several existing schemes of optical buffering, including fixed fibre delay lines ${ }^{1}$, and the use of resonators ${ }^{2}$. However, there are some major drawbacks in these schemes. Fixed delay lines are not flexible as they can only offer fixed delay times and their size can be bulky. For optical resonators, although they could offer significant optical delay in a small package, their operating bandwidth is typically very narrow $(\sim 1 \mathrm{~nm})^{3}$. In this paper, we present the design and fabrication of an optical buffer device based on two suspended parallel waveguides using the indium phosphide (InP) platform. The theoretical investigation of such optical buffer design in silicon has been reported in our previous work ${ }^{4}$, and here we investigate such optical buffer realization in III-V semiconductor material. III-V materials such as InP and GaAs have some significant advantages over silicon. The direct band gap nature of III-V materials means that optical gain can be achieved. Other optical components such as amplifiers and lasers are well established in the III-V platform ${ }^{5}$, hence our device can be easily integrated in the existing infrastructure. Another advantage of our design is that the predicted bandwidth of operation is very wide $(\sim 100 \mathrm{~nm})$ and covers the whole C-band of communication wavelengths. 


\section{OPTICAL BUFFER DESIGN}

Our optical buffer design is based on two parallel waveguides with sub-micron cross section dimensions and they are suspended in air. Due to their small dimensions, the optical mode is confined in both the waveguides and the spacing between them, rather than guiding in the individual waveguides. The schematic geometry of our optical buffer design is shown in Figure 1.

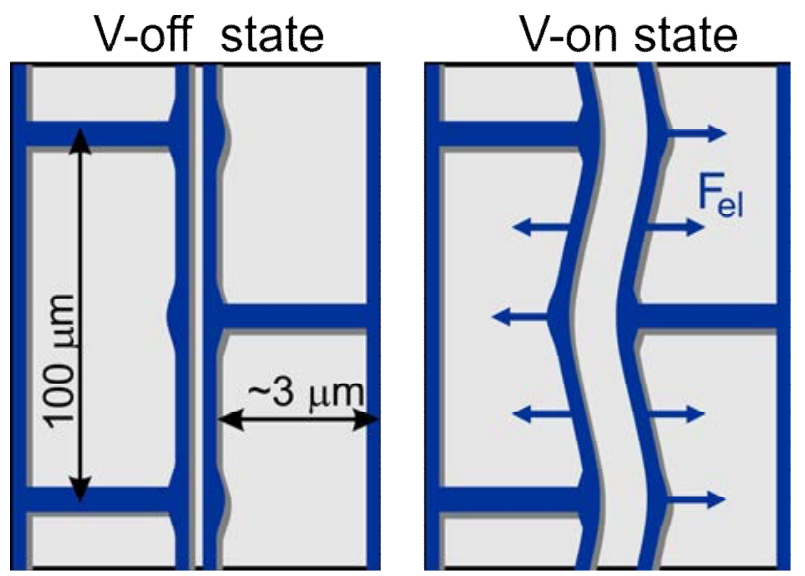

Figure 1. Schematic diagram showing the geometry of the parallel waveguides optical buffer design (top view, not to scale) with and without the applied voltage. The waveguides are fixed at the pillars, and the waveguides are pulled apart by the electrostatic repulsion ( $\mathrm{F}_{\mathrm{el}}$, indicated by the arrows).

The way we physically achieve the variation of air gap size is by MEMS actuation. The method that we discuss here is an "active" actuating method. This relies on voltages applied directly into the waveguide layer of the structure. In the proposed scheme, the waveguides are actuated by electrostatic repulsive forces, created by applying the same polarity of voltage to both waveguides and grounding the bottom substrate $(\sim 4 \mu \mathrm{m}$ below the waveguides). This deforms the waveguides and increases the air gap between them (as schematically shown in Figure 1). Actuation with repulsive forces has an advantage over actuation with attractive forces: the pull-in instability causing waveguide sticking is avoided.

We modelled the waveguide mechanical deformation caused by electrostatic repulsive forces. Firstly, the repulsive force between the waveguides was calculated depending on both the applied voltage and the air gap using a finite element method. After that, the mechanical deformation of a waveguide suspended between two support pillars caused by the electrostatic force distributed along the waveguide length was calculated. A distance between the pillars of $100 \mu \mathrm{m}$ was assumed. This provided sufficient flexibility of the waveguides and enabled a mechanical oscillation frequency of the order of $100 \mathrm{kHz}$. The alternating position of the pillars (shown in Figure 1) ensured minimal variation of the air gap in the actuated device. The initial air gap between the waveguides was designed to be $50 \mathrm{~nm}$.

The spacing between the waveguides is key to the tunability of the optical delay achieved in our buffer design. When the air gap is small, i.e. the waveguides are close together, a larger fraction of the optical signal propagates inside the waveguides, and this leads to a higher effective group index. On the other hand, when the air gap increases, the optical signal is less confined in the waveguides and spreads into the air between them, and this leads to a lower effective group index. 


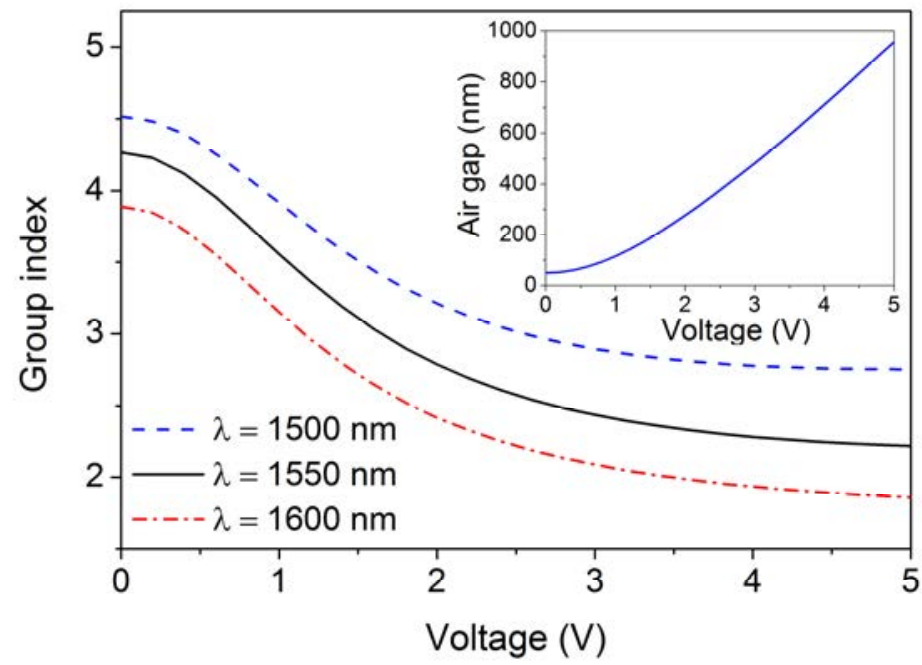

Figure 2. Group index of the symmetric TE supermode as a function of the air gap between coupled waveguides. Inset: Achievable air gap size vs actuation voltage applied in our design.

In our previous investigation ${ }^{4}$, we predicted that we could maximize the group index contrast for the TE mode by having individual waveguide dimensions of $195 \mathrm{~nm}$ by $300 \mathrm{~nm}$. Figure 2 shows the corresponding group index variation of the propagating symmetric TE mode dependent on voltage for three different wavelengths in the spectral range from 1500 $\mathrm{nm}$ to $1600 \mathrm{~nm}$. Most of the group index variation (and hence the propagation delay) is observed within a voltage range from 0 to $3 \mathrm{~V}$, which corresponds to an air gap change from $50 \mathrm{~nm}$ to $500 \mathrm{~nm}$. A variation of the signal propagation time of $\sim 10 \mathrm{ps}$ is expected in a $1 \mathrm{~mm}$ long device. The air gap averaged over the device length depending on the actuation voltage is shown in the inset in Figure 2. An air gap variation form $50 \mathrm{~nm}$ to $1000 \mathrm{~nm}$ with $5 \mathrm{~V}$ applied to the electrodes is predicted.

\section{FABRICATION}

The III-V MEMS optical buffer structure was grown on an InP substrate by molecular beam epitaxy (MBE). A $4 \mu \mathrm{m}$ InGaAs layer was grown first on the substrate; this layer served as a sacrificial layer. Subsequently, a $300 \mathrm{~nm} \mathrm{InGaP}$ device layer was grown on top of the InGaAs layer. A schematic diagram of the MBE grown structure is shown in Figure 3. In the InGaP layer, the Ga content was only $1 \%$, its purpose being to introduce tensile stress into the device layer and help to avoid waveguide collapse after the waveguide release step. The device layer was n-doped with a carrier concentration of $1 \times 10^{17} \mathrm{~cm}^{-3}$ to enable electrically conductive waveguides as discussed in the previous section.

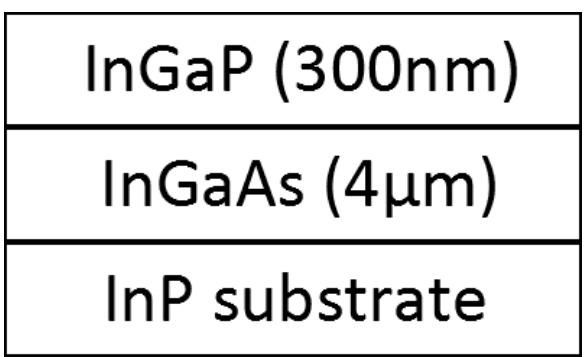

Figure 3. Schematic diagram of the layer structure of the optical buffer device. 
During the MBE growth, it is important to achieve layer surfaces as smooth as possible in order to minimize optical scattering losses when the optical signal is travelling though the device. In order to analyze the surface roughness at the interface between the InGaAs and InGaP layer, a $4 \mu \mathrm{m}$ InGaAs layer was grown under the same growth condition as the optical buffer structure. The surface profile of the InGaAs surface was measured under tapping mode using an atomic force microscope (Bruker Icon). A 3D AFM topographic image of the surface is shown in Figure 4. Results revealed that the InGaAs surface was very smooth. The r.m.s. value of the surface roughness was $0.376+/-0.04 \mathrm{~nm}$, with typical maximum height difference of $3.2+/-0.4 \mathrm{~nm}$.

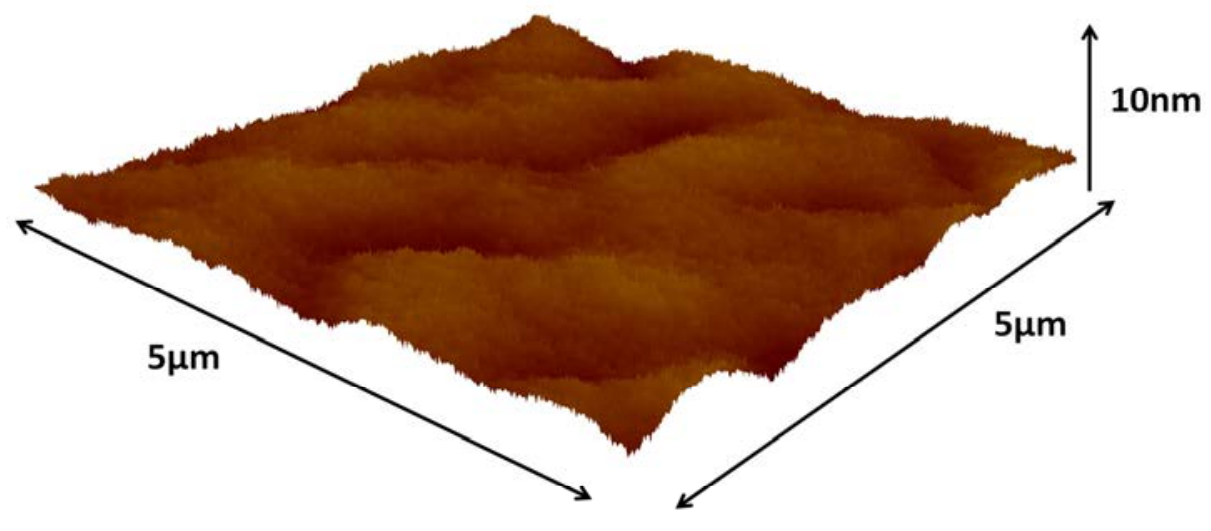

Figure 4. 3D AFM topographic image of the MBE grown InGaAs sacrificial layer surface measured in tapping mode.

The optical buffer device was fabricated on a $1 \mathrm{~cm}$ by $1 \mathrm{~cm}$ chip. The fabrication process started by patterning the electrodes for electrostatic actuation by electron beam lithography. Gold electrodes were deposited by thermal evaporation, followed by lift off in acetone.

After the gold electrodes were deposited on the device layer, the waveguides and pillar supports were patterned again by electron beam lithography; HSQ was used as a negative tone electron beam resist. In the first generation of device, the dimensions of the written waveguide were $250 \mathrm{~nm}$ in width and $200 \mu \mathrm{m}$ in length. The pillar supports were patterned in the same step, and the distance between supports was set to be $40 \mu \mathrm{m}$ apart. Each pillar section consisted of two parts, an S-shaped part attached to the waveguide, and a rectangular section that attached the S-shaped part to the large contact pad. The air gap between the waveguide was approximately $300 \mathrm{~nm}$. The distance between the waveguides and the contact pads was $3 \mu \mathrm{m}$. An SEM image of a section of the patterned waveguides and pillar supports is shown in Figure 5 a. 

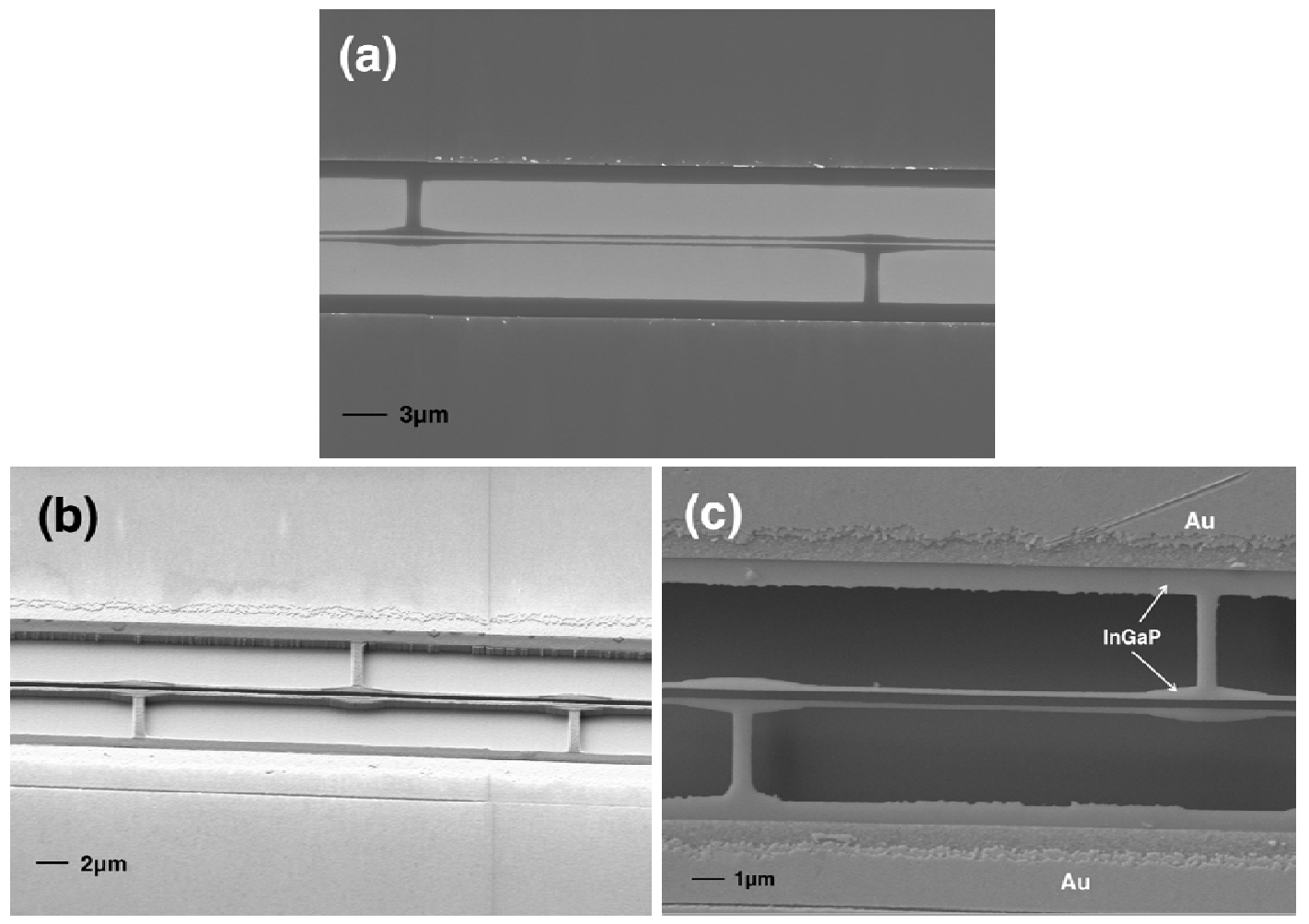

Figure 5. SEM image showing (a) a section of the patterned waveguide and pillar by electron beam lithography; (b) the waveguide and pillar structure after a reactive ion etch; (c) after waveguide release and supercritical drying.

After the electron beam patterning, the structure was etched by reactive ion etching, using a methane and hydrogen plasma. This etch chemistry provided an excellent anisotropic etch with the sidewall angle better than 80 degrees, as shown in Figure 5b. After the RIE etch, the waveguides and pillar supports were released using an HF based solution. This etch has very good selectivity between InGaAs and InGaP. The SEM image in Figure 5c shows the InGaAs layer was etched cleanly, without leaving any residual resist on the structure. In order to avoid stiction, the release structure was dried using $\mathrm{CO}_{2}$ supercritical drying.

The fabricated waveguide structure has dimensions of approximately $300 \mathrm{~nm}$ (height) by $200 \mathrm{~nm}$ (width) and $200 \mu \mathrm{m}$ (length). These dimensions were comparable with our design. However, the air gap was $400 \mathrm{~nm}$ wide, which was larger than we patterned. This difference is due to the sidewall etching of the waveguides during RIE. This can be rectified by adjusting the size and position of the waveguides in the initial electron beam patterning step.

\section{CONCLUSION}

We have demonstrated the design and fabrication of an InP parallel waveguide structure that is designed for use as an optical buffer. The air gap between the waveguides is designed to be adjustable by MEMS actuation. Our simulation shows a $100 \%$ change in propagation delay is predicted by varying the air gap size from $50 \mathrm{~nm}$ to $500 \mathrm{~nm}$, and a propagation delay of $10 \mathrm{ps}$ is predicted with a $1 \mathrm{~mm}$ long device. In our first generation of fabricated device, each waveguide has a transverse dimension of $200 \mathrm{~nm}$ x $300 \mathrm{~nm}$, and the spacing between the waveguides was $400 \mathrm{~nm}$. The air gap was significantly larger than designed but this could be reduced by offsetting the lithography pattern. Future work will focus on optimizing the fabricated device dimensions and to demonstrate its functionality as an optical buffer. 


\section{REFERENCES}

[1] Langenhorst, R., Eiselt, M., Pieper, W., Großkopf, G., Ludwig, R., Küller, L., Dietrich, E. and Weber, H. G., "Fiber loop optical buffer," J. Lightw. Technol. 14, pp. 324-335 (1996).

[2] Xia, F., Sekaric, L. and Vlasov Y., "Ultracompact optical buffers on a silicon chip,” Nature Photon. 1, pp. 65-71 (2007).

[3] Poon, J. K. S., Zhu, L., DeRose, G. A. and Yariv, A., "Transmission and group delay of microring coupledresonator opticalwaveguides," Opt. Lett. 33, pp. 456-458 (2006).

[4] Horak, P., Stewart, W. and Loh, W. H., "Continuously tunable optical buffer with a dual silicon waveguide design,” Opt. Exp. 19, pp. 12456-12461 (2011).

[5] Lelange F., Bagens Lelarge, B., Renaudier, J., Brenot, R., Accard, A., van Dijk, F., Make, D., Le Gouezigou, O., Provost, J.-G., Poingt, F., Landreau, J., Drisse, O., Derouin, E., Rousseau, B., Pommereau, F., Duan, G. H., "Recent advances on InAs/InP quantum dash based, semiconductor lasers and optical amplifiers operating at

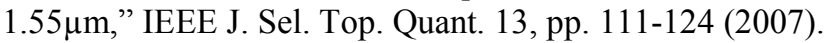

